\title{
The involvement of HER2 and p53 status in the regulation of telomerase in irradiated breast cancer cells
}

\author{
VASSILIS PAPANIKOLAOU ${ }^{1,2}$, DIMITRIOS ILIOPOULOS ${ }^{3}$, IOANNIS DIMOU ${ }^{4}$, STEPHANIE DUBOS ${ }^{2}$, \\ IOANNIS TSOUGOS ${ }^{5}$, KYRIAKI THEODOROU ${ }^{5}$, SOFIA KITSIOU-TZELI ${ }^{6}$ and ASPASIA TSEZOU ${ }^{1,2,7}$ \\ ${ }^{1}$ Laboratory of Cytogenetics and Molecular Genetics, Medical School, University of Thessaly, 41110 Larissa; \\ ${ }^{2}$ Institute for Biomedical Research and Technology, 41222 Larissa, Greece; ${ }^{3}$ Department of Molecular Virology, \\ Immunology and Medical Genetics, The Ohio State University Comprehensive Cancer Center, Columbus, OH 43210, USA; \\ ${ }^{4}$ E.C.E. Department, Technical University of Crete, 73100 Chania; ${ }^{5}$ Department of Medical Physics, Medical School, \\ University of Thessaly, 41110 Larissa; ${ }^{6}$ Department of Medical Genetics, Medical School, University of Athens, \\ 11527 Athens; ${ }^{7}$ Department of Biology, Medical School, University of Thessaly, 41110 Larissa, Greece
}

Received February 11, 2009; Accepted March 27, 2009

DOI: 10.3892/ijo_00000430

\begin{abstract}
Cancer cell characteristics may play a pivotal role in the response to therapy by activating or deactivating different molecular pathways. In the present study, we investigated the implication of breast cancer cell features, such as HER2 and p53 in the activation of telomerase upon exposure to ionizing radiation. Telomerase is among the most important cancer biomarkers, conferring to tumor cells unlimited proliferative capacity, increased survival potential and resistance to several types of cellular stress. We investigated possible mechanisms regulating telomerase in six irradiated breast cancer cell lines (MCF-7, MCF-7/HER2, MDA-MB-231, SK-BR-3, BT-474 and HBL-100) differing in their HER2, p53 and ER $\alpha$ status. hTERT mRNA expression was evaluated by real-time PCR and telomerase activity by the TRAP assay. HER2, c-myc, p53 and p21 protein levels were evaluated by Western blotting. Silencing of hTERT and HER2 was achieved by small interfering RNA technology. Chromatin immunoprecipitation was used to evaluate $\mathrm{H} 3$ histone acetylation status, as well as $\mathrm{myc} / \mathrm{mad} / \mathrm{max}$ and $\mathrm{p} 53$ transcription factors interaction with the $h T E R T$ promoter. Our results showed for the first time, that only HER2-positive cells, independently of their p53 status, upregulated hTERT/telomerase, while knockdown of hTERT increased radio-sensitivity. Knockdown of HER2 also led to increased radio-sensitivity and downregulation of hTERT/ telomerase. We also demonstrated that c-myc and mad1 regulate hTERT expression in all irradiated breast
\end{abstract}

Correspondence to: Dr Aspasia Tsezou, Department of Biology, Medical School, University of Thessaly, Mezourlo, 41110 Larissa, Greece

E-mail: atsezou@med.uth.gr

Key words: HER2, ionizing radiation, breast cancer, telomerase, p53 cancer cells. We conclude, for the first time, that HER2 phenotype upregulates hTERT through c-myc activation and confers radio-resistance to breast cancer cells.

\section{Introduction}

Breast cancer is the most common malignancy among females in most Western countries, with an overall lifetime risk of $10 \%$ of developing invasive breast cancer (1). Ionizing radiation plays a key role in the treatment of breast cancer, however, intrinsically resistant to ionizing radiation tumors pose significant limitations to radiotherapy (2). Among factors that contribute to tumor radioresistance are deprivation of essential growth factors, blockade of growth factor receptors, as HER2, or activation of genes involved in cell cycle arrest, DNA repair and apoptosis $(3,4)$.

The clinical efficacy of radiotherapy may be assessed by molecular biomarkers, which constitute targets for the diagnosis of malignancy (5-7). The observation that telomerase activity is present in 85 to $90 \%$ of all human tumors, but not in normal non-neoplastic cells $(5,6,8)$ has made telomerase a marker of malignancy and a target not only for the diagnosis of malignancy, but also for the development of novel therapeutic agents (9). Telomerase, is a ribonucleoprotein complex that extends and maintains telomeres, and activation of this enzyme is therefore required for cells to overcome replicative senescence and obtain the ability to divide without limits $(10,11)$. Recently it was shown that telomerase consists of two molecules of each telomerase reverse transcriptase (hTERT), telomerase RNA and dyskerin (12). However, the key component for the control of telomerase activity is the catalytic hTERT subunit (13-15).

It has been shown that ionizing radiation may have the potential to reduce telomerase activity, reducing therefore the proliferative capacity of tumor cells. Furthermore, specific changes of telomerase activity could be used as a predictive parameter of radiation outcome and a monitor of the overall effects of radiation (16). Telomerase enzymatic activity can be regulated at multiple levels, including hTERT transcription, 
alternative splicing, chaperone-mediated folding, phosphorylation and nuclear translocation; however, the major control mechanism of telomerase regulation seems to be at the level of hTERT transcription (17). Different features of tumor cells such as HER2, ER $\alpha$ and p53 may be responsible for the regulation of telomerase, when these are treated by irradiation or chemotherapy agents. hTERT expression has been found to correlate with the overexression of HER2 gene. More specifically, in breast cancer, hTERT expression has been found increased in HER2-positive breast tumors and breast tumor cell lines, compared to their HER2-negative counterparts (18). On the other hand, p53 has been shown to directly interact with the transcription machinery and to negatively regulate a variety of cellular genes (19). The fact that hTERT/telomerase and p53 seem to counteract each other in the context of cellular aging and immortalization and that the activation of telomerase and inactivation of p53 are characteristic of human tumors raises the question of whether there exists a link between hTERT/telomerase regulation and p53 (9).

In the present study, we investigated the involvement of HER2 and p53 status in hTERT/telomerase regulation in irradiated breast cancer cells.

\section{Materials and methods}

Cell cultures. The breast cancer cell lines used were: MCF-7 (p53 wild-type, ER $\alpha$-positive, weak for HER2), MCF-7/HER2 (p53 wild-type, ER $\alpha$-positive, constitutively expressing HER2), MDA-MB-231 (p53 mutant, ER $\alpha$-negative, weakly positive for HER2), HBL100 (p53 wild-type, ER $\alpha$-negative, weak for HER2), SK-BR-3 (p53 mutant, ER $\alpha$-negative, HER2-positive) and BT-474 (p53 thermosensitive mutant, acting as mutant when cells are grown at $37^{\circ} \mathrm{C}, \mathrm{ER} \alpha$-positive, HER2-positive). All cell lines were cultured in RPMI-1640 medium (Gibco, Paisley, UK) supplemented with $10 \%$ fetal bovin serum (Gibco), L-glutamine $2 \mathrm{mM}$ (Gibco), penicillin $100 \mathrm{IU} / \mathrm{ml}$ and streptomycin $100 \mu \mathrm{g} / \mathrm{ml}$ (Gibco), at $37^{\circ} \mathrm{C}$ in $5 \% \mathrm{CO}_{2}$.

Irradiation. Cells in exponential growth were irradiated with 10 and 20 Gy doses of ionizing radiation, using a $6 \mathrm{MV}$ photon linear accelerator with a dose rate of $4.90 \mathrm{~Gy} / \mathrm{min}$ at room temperature. Ten and 20 Gy doses were chosen based on our previous dose titration data (20). Two separate experiments were carried out for each cell line studied. In order to ensure a uniform dose build-up and homogeneous irradiation cells were situated at the isocenter of two tangential opposed photon fields of 20x20 field size. For the accurate positioning and immobilization of the culture plates and flasks and to ensure reproducibility and accurate doses of irradiation, a homogeneous polymethyl methacrylate $\mathrm{C}_{5} \mathrm{H}_{8} \mathrm{O}_{2}$ (PPMA) phantom was constructed, as previously described by us (20).

Cell viability. Cell viability was determined with the MTT assay using the TACS MTT kit (R\&D Systems, Minneapolis, MN, USA) according to manufacturer's instructions. The MTT cell proliferation and viability assay is an in vitro assay for the measurement of cell proliferation or reduction of cell viability, when metabolic events lead to apoptosis or necrosis.
hTERT quantitative mRNA expression. Total RNA was extracted using TRIzol reagent according to manufacturer's instructions (Gibco). Preservation of $28 \mathrm{~S}$ and $18 \mathrm{~S}$ rRNA species was used to assess RNA integrity. Only samples with prominent $28 \mathrm{~S}$ and $18 \mathrm{~S}$ rRNA components were included in the study. Total RNA was reversed transcribed to cDNA using SuperScript first-strand synthesis (Invitrogen Carlsbad, CA, USA) for reverse transcription-PCR using the oligo(dT) primer according to manufacturer's instructions. Real-time RT-PCR for hTERT, was performed using FastStart Universal SYBRGreen Master (ROX) (Roche, Mannheim, Germany) in a iCycler Optical Module (Bio-Rad, Hercules, CA). Reactions were performed in triplicate using $2 \mu 1$ of cDNA per reaction and primers specific for hTERT (forward: 5'-CCG TCT GCG TGA GGA GAT-3'; reverse: 5'-TGG GGA TGA AGC GGA GTC-3'), while human porphobilinogen deaminase (PBGD) was used as a housekeeping gene (forward: 5'-AGA GTG ATT CGC GTG GGT ACC-3'; reverse: 5'-GGC TCC GAT GGT GAA GCC-3').

Telomeric repeat amplification protocol (TRAP). TRAP assay is considered a sensitive and specific PCR-based functional enzyme assay. It was performed using the TeloTAGGG telomerase PCR ELISA PLUS kit (Roche, Indianapolis, IN, USA), as previously described (21).

Western blot analysis. Irradiated and non-irradiated cells were trypsinized, collected and centrifuged for $7 \mathrm{~min}$ at 2,000 rpm. Cell pellets were lysed using Nonidet P-40 lysis buffer containing $30 \mathrm{mM}$ Tris (pH 7.5), $150 \mathrm{mM} \mathrm{NaCl}, 10 \%$ glycerol, $1 \%$ Nonidet P-40 and a cocktail of protease inhibitors for $30 \mathrm{~min}$ on ice, followed by centrifugation for $20 \mathrm{~min}$ at $12,000 \mathrm{rpm}$. Supernatants were transferred in new tubes and stored at $-80^{\circ} \mathrm{C}$. Protein concentration was quantified using the Bio-Rad Bradford protein assay, with bovine serum albumin as standard. Equal amounts of protein were electrophoresed and separated by $10 \%$ SDS-PAGE (Bio-Rad) and transferred to a Hybond-ECL nitrocellulose membrane (Amersham Biosciences, Piscataway, NJ). The membrane was incubated with specific antibodies to c-myc (sc-764), p53 (sc-6243) and p21 (sc-397) (sc-10811, Santa Cruz Biotechnology, CA, USA), HER2 (MS-441-S) (Thermo Fisher Scientific, UK) $(1: 800)$ and signals were detected using anti-rabbit immunoglobulin IgG conjugated with horseradish peroxidase $(1: 5,000)$. The chemiluminescence was resolved by an enhanced chemiluminescence ECL kit (Amersham, Milan, IT). The results were normalized by anti-actin monoclonal antibody.

Chromatin immunoprecipitation. Chromatin immunoprecipitation was performed using a ChIP assay kit (Upstate USA, Inc., Charlottesville, VA, USA) on irradiated and non-irradiated cells. Briefly, cells were cross-linked by incubating them in $1 \%$ ( $\mathrm{vol} / \mathrm{vol})$ formaldehyde-containing medium for $10 \mathrm{~min}$ at $37^{\circ} \mathrm{C}$ and then sonicated to make soluble chromatin with DNA fragments between 200 and 1000 bp. Samples of total chromatin were taken at this point to use as a positive control in the PCRs (input chromatin). The cell lysates were precleared by incubation with G-Sepharose beads and then incubated with the polyclonal antibodies [anti-max 
(sc-197), anti-c-myc (sc-764), anti-mad1 (sc-222), anti-p53 (sc-6243)] (Santa Cruz Biotechnology) overnight at $4^{\circ} \mathrm{C}$. For analysis of acetylation status, antibodies against acetylated histone H3 (06-599) (Upstate Biotechnology, Lake Placid, NY) were used for immunoprecipitation. DNA-protein complexes were collected with G-Sepharose beads followed by several rounds of washing, eluted and reverse crosslinked. Following treatment with Protease K (Sigma), the samples were extracted with phenol-chloroform and precipitated with ethanol. The recovered DNA was resuspended in TE buffer and used for the PCR amplification. The primer sequences used for $h T E R T$ promoter were (forward: 5'-TGT CGG GGC TAG GCC GGG CTC-3'; reverse: 5'-AAC TCG CGC CGC GAG GAG A-3'). The PCR products were fractionated on $3 \%$ agarose gels and stained with ethidium bromide.

Knockdown of hTERT and HER2 mRNA expression. Cells were transfected with dsRNA oligonucleotides for hTERT [1442470148 (Invitrogen)] and HER2 [s65633 (Ambion, TX, USA)] using Lipofectamine 2000 reagent (Invitrogen) according to manufacturer's instructions. Cells were cultured in Optimem medium (Invitrogen) in 96-well plates for $24 \mathrm{~h}$ in order to achieve a $50 \%$ confluence by the time of tranfection.

Statistical analysis. Statistical significance for mRNA expression analysis, was determined using Student's t-test using a confidence level of $95 \%(\mathrm{P}<0.05)$. All experiments were performed in triplicate.

The effect of siRNA against HER2 on HER2 and hTERT mRNA expression, as well as on telomerase activity, and of siRNA against hTERT on hTERT mRNA expression and telomerase activity, was measured utilizing single factor analysis of variance. F-statistics was used to quantify the investigated effects showing the amount of overall variance that is induced due to the knockdown effect.

In order to statistically evaluate the combined effects of the HER 2 or hTERT knockdown on cell viability, along with the irradiation process, we employed 2-factor analysis of variance. Cell viability was measured and evaluated against siRNA for HER2 or hTERT and irradiation at three timepoints $(24,48,72 \mathrm{~h})$. The time of measurement was incorporated in the model as an additional covariate to account for the corresponding variance component. Additionally, confidence intervals were estimated for all measurement sets to visualize experimental variability using Student's t-distribution, to account for the limited number of samples.

\section{Results}

Ionizing radiation causes different effects on hTERT/ telomerase in the different breast cancer cell lines studied. hTERT mRNA expression levels and telomerase activity were evaluated in all the cell lines studied. It was observed that HER2-positive cell lines (MCF-7/HER2, SK-BR-3 and BT-474) exhibited significantly higher hTERT/telomerase levels than HER2-negative (MCF-7, MDA-MB-231 and HBL100) cells (Fig. 1A and B).
After irradiation, we observed that MCF-7, MDA-MB-231 and HBL100 cells demonstrated a significant $(\mathrm{P}<0.05)$ downregulation in hTERT mRNA expression, which was correlated with a reduction in telomerase activity, with the exception of MCF-7 cells at $24 \mathrm{~h}$. On the contrary, irradiated SK-BR-3 and BT-474 cells, demonstrated an increase in both hTERT expression and telomerase activity. Additionally, MCF-7/HER2 cells showed a different profile than the parental MCF-7 cells, exhibiting an initial hTERT/telomerase downregulation followed by upregulation at $72 \mathrm{~h}$ post-irradiation and afterwards $(\mathrm{P}<0.05)$.

Implication of HER2 in the regulation of hTERT/telomerase. The observations that HER2-positive cells (SK-BR-3 and BT-474) exhibited upregulation of hTERT/telomerase after irradiation compared to HER2-negative cells (MCF-7, MDAMB-231 and HBL100), showing hTERT/telomerase downregulation, along with the difference in hTERT/telomerase profile after irradiation of MCF-7 and MCF-7/HER2 cells prompted us to investigate the implication of HER2 status in hTERT expression following irradiation of breast cancer cells. We studied at first HER2 protein expression levels in all breast cancer cell lines (Fig. 2A). As expected, weakly expressing HER2 cells (MCF-7, MDA-MB-231 and HBL-100), showed low HER2 levels with no significant modification after irradiation, while HER2-positive (SK-BR-3, BT-474 and MCF-7/HER2) cells showed high HER2 levels which were upregulated after irradiation.

In order to further investigate the implication of HER2 status in the regulation of hTERT, we knocked down HER2 expression in HER2-positive SK-BR-3 cells using siRNA technology and liposomal transfection. The estimated mean values of HER2 and hTERT mRNA expression, as well as of telomerase activity and the corresponding confidence intervals are given in Fig. 2. We observed a dose-dependent decrease of HER2 mRNA (panel C) and protein (panel B) expression followed by an analogous decrease in hTERT mRNA expression levels and telomerase activity at $24 \mathrm{~h}$ post-transfection (panel C). The associated F-statistics indicated a strong influence of siHER2 on HER2 ( $\mathrm{F}=205.4$, $\mathrm{P}<0.05)$ and hTERT $(\mathrm{F}=239.5, \mathrm{P}<0.05)$ mRNA expression.

c-myc, p53 and p21 protein levels differ in the different breast cancer cell lines following irradiation. We also evaluated c-myc, p53 and p21 protein levels after irradiation in the six breast cancer cell lines (Fig. 3). We found that c-myc protein levels were high only in non-irradiated SK-BR-3 and BT-474 cell lines and were upregulated in SK-BR-3, BT-474 and MCF-7/HER2 cells after irradiation. MCF-7, MDA-MB231 and HBL100 cells demonstrated very low c-myc protein expression after irradiation.

We then examined p53 protein levels in both p53 wild-type (MCF-7, MCF-7/HER2 and HBL100) and p53 mutant (MDA-MB-231, SK-BR-3 and BT-474) cells post-irradiation, as well as the ability of p53 to induce p21 in these cell lines. We found that wild-type and mutant p 53 were upregulated in all cell lines upon irradiation (with the exception of BT-474 cells). We also found that the ability of p53 to induce p21 differed between the cell lines studied. Wild-type $\mathrm{p} 53$ protein up-regulated significantly p21 in MCF-7, MCF-7/HER2 and 
A

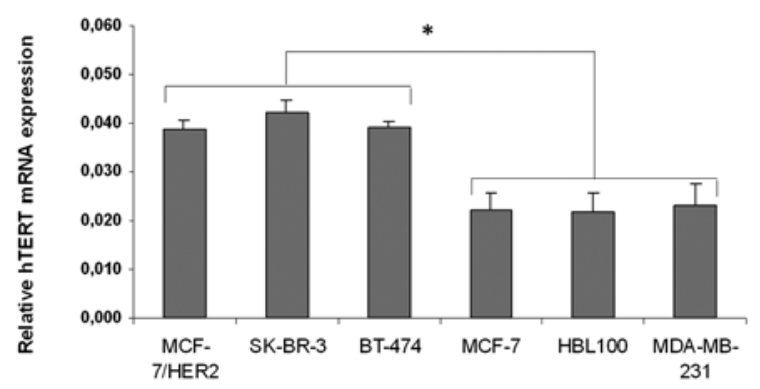

C

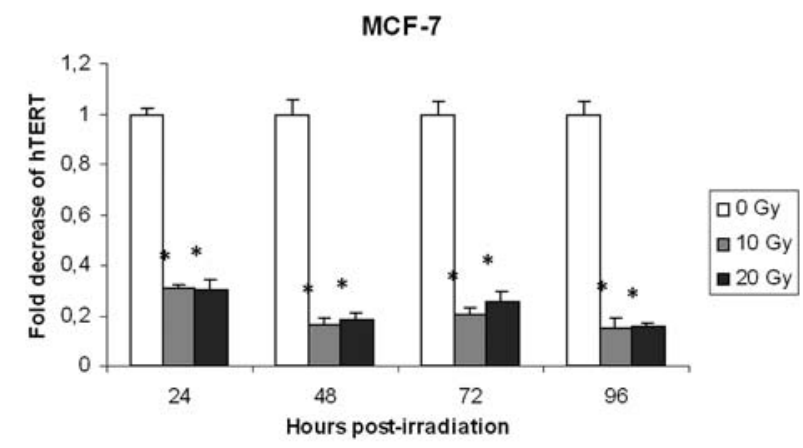

MCF-7/HER2

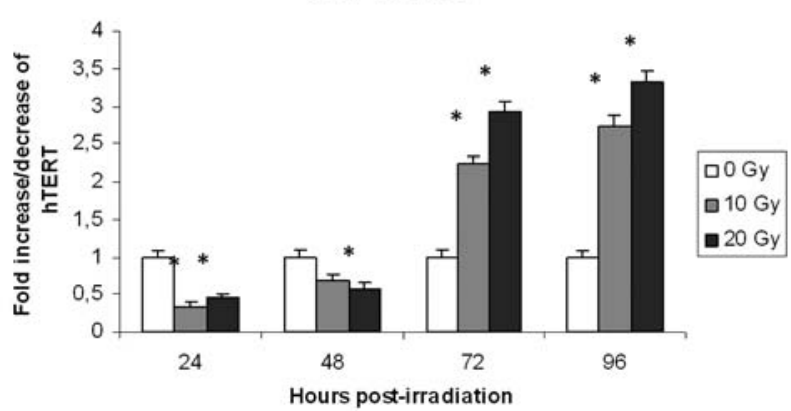

HBL100

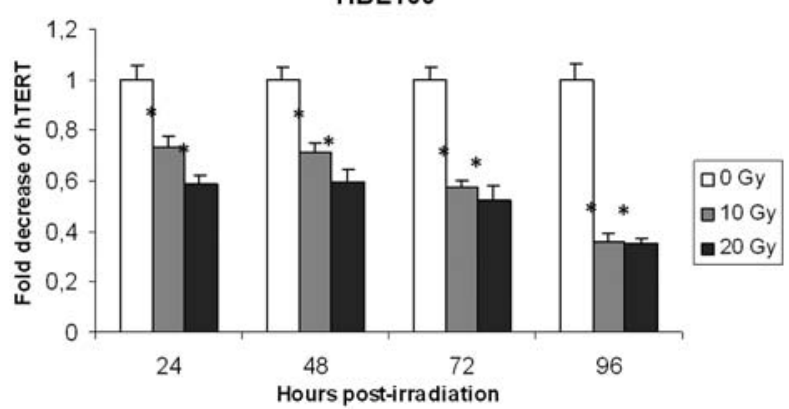

B

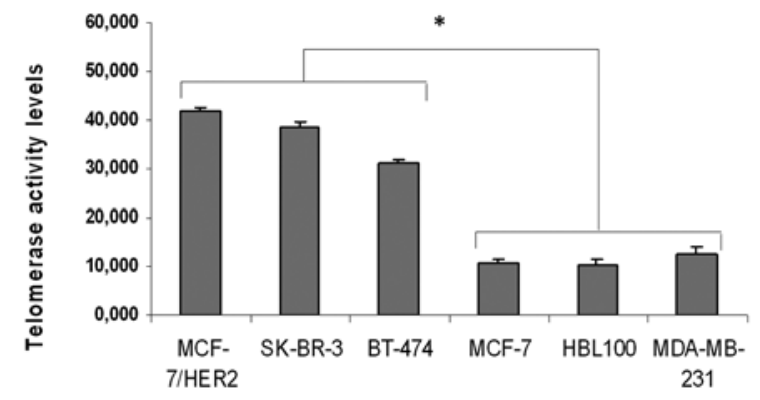

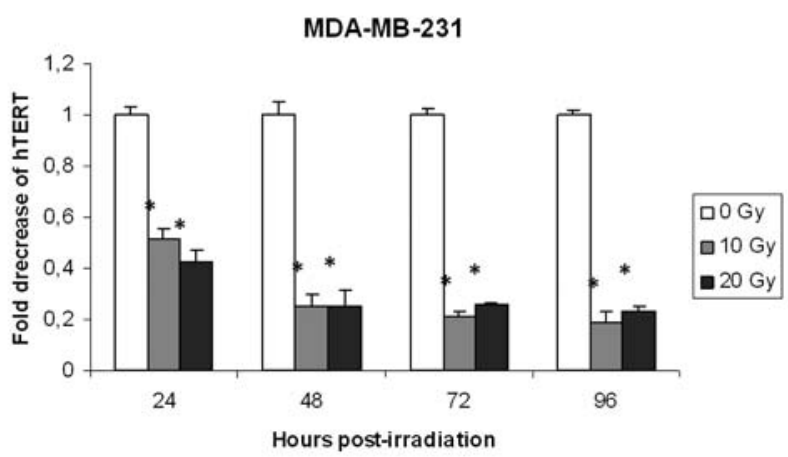
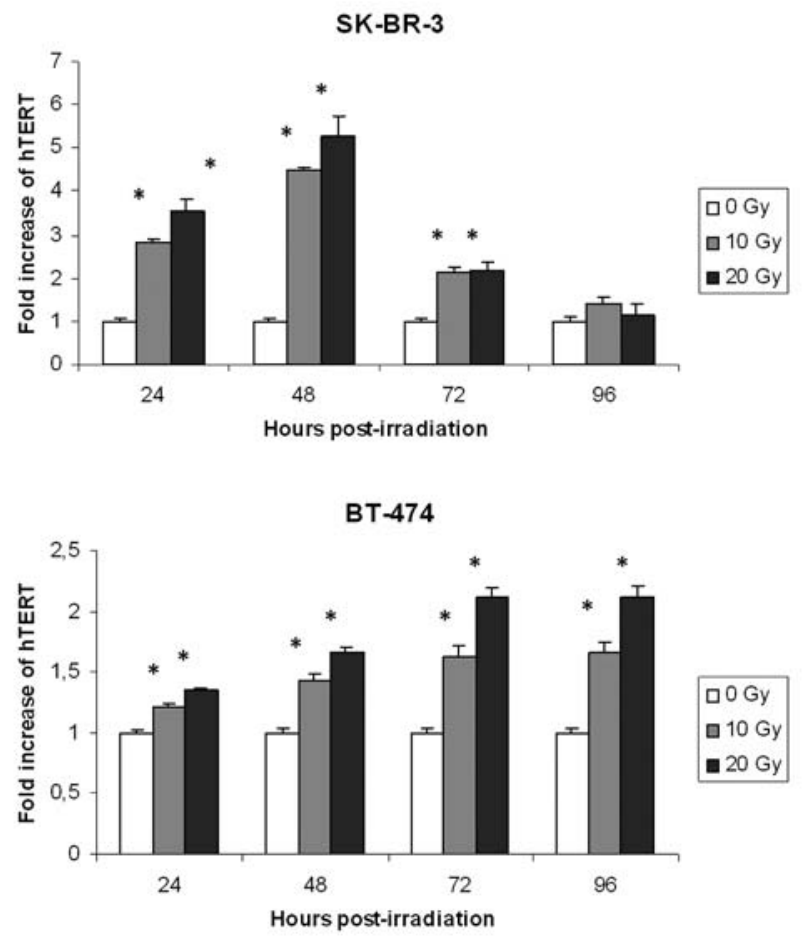

Figure 1. hTERT mRNA expression levels, normalized to expression levels of the PBGD housekeeping gene and telomerase activity levels were compared between non-irradiated and irradiated breast cancer cell lines at 24, 48, 72 and $96 \mathrm{~h}$. (A) Comparison of hTERT mRNA levels between non-irradiated breast cancer cell lines. (B) Comparison of telomerase activity levels between non-irradiated breast cancer cell lines. (C) hTERT mRNA expression, upon irradiation of breast cancer cell lines. Histograms represent the fold increase or decrease of hTERT expression in irradiated cells, compared to non-irradiated cells. The normalized hTERT expression of non-irradiated cells was set to 1 .

HBL100 cells, while mutant p53 protein upregulated p21 in MDA-MB-231 and SK-BR-3 cells. p21 protein was not detected in BT-474 cells.

Chromatin immunoprecipitation assay: correlation of hTERT mRNA expression profile with a switch of myc/max or mad/max complexes in its promoter. As illustrated in Fig. 4, a strong signal was observed in the max immunoprecipitation in all six breast cancer cell lines, while no signal was observed when anti-p53 was used to precipitate chromatin. In order to verify the specificity of the procedure, precipitated DNA from p53 samples, was subjected to PCR for the $p 21$ promoter and 

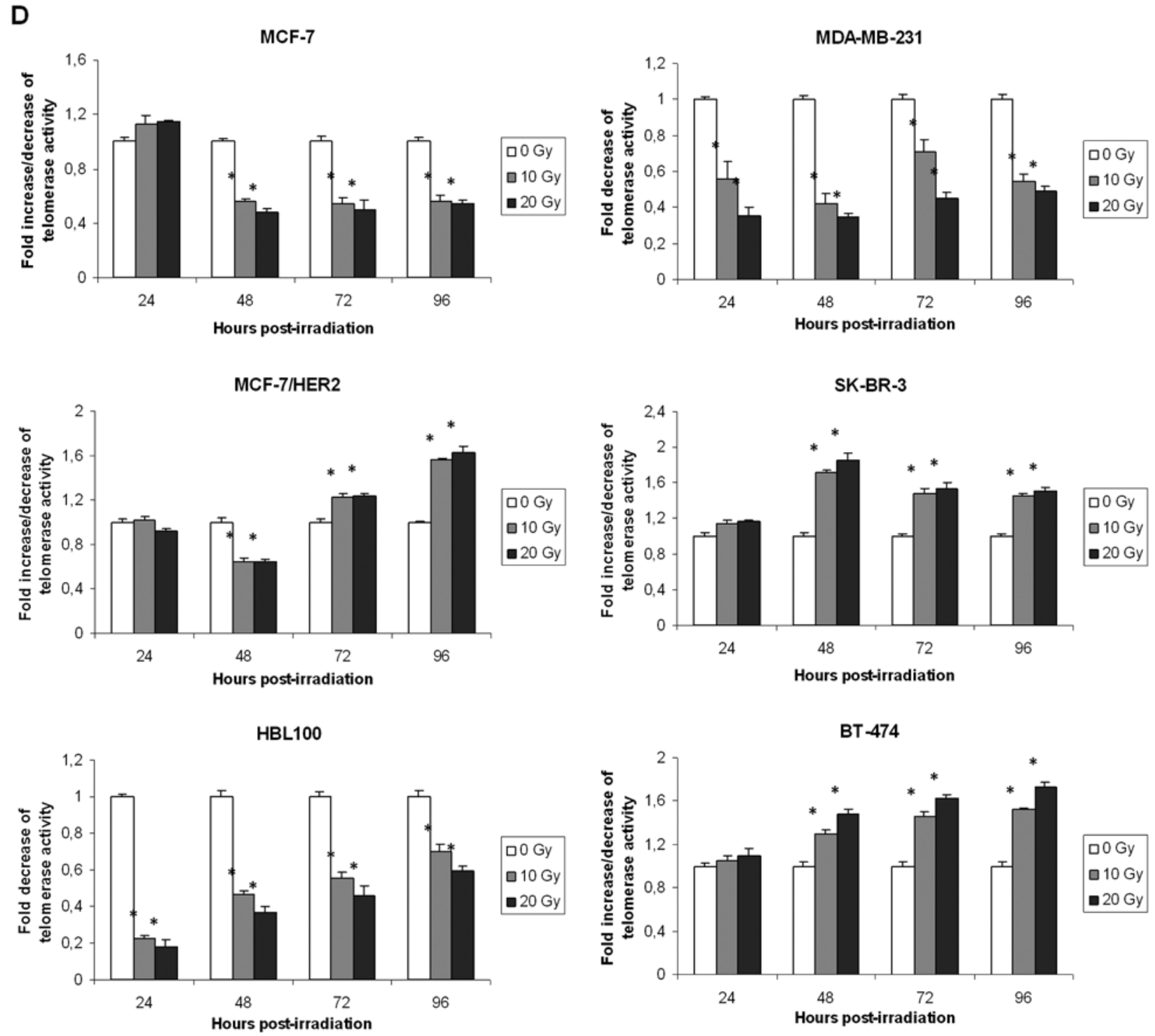

Figure 1. (D) Histograms represent the fold increase or decrease of telomerase activity in irradiated cells, compared to non-irradiated cells. The normalized telomerase activity of non-irradiated cells was set to 1 . Bars represent standard errors and asterisks designate statistically significant results $(\mathrm{P}<0.05)$.

samples were positive for all cell lines except BT-474 (data not shown). Acetylated $\mathrm{H} 3$ histone was present in all irradiated and non-irradiated samples, but we observed a stronger signal in non-irradiated samples of MCF-7, MDA-MB-231 and HBL100 cells in comparison to their irradiated counterparts. The inverse was observed for MCF-7/HER2 cells where a stronger signal for $\mathrm{H} 3$ precipitated samples was observed in irradiated than in non-irradiated cells (no such difference was observed for SK-BR-3 and BT-474 cell lines).

Regarding the $\mathrm{myc} / \mathrm{mad} / \mathrm{max}$ protein complex, we observed a strong signal for both c-myc and mad1 precipitations in non-irradiated cells, demonstrating heterogeneity in the cell population. MCF-7, MDA-MB-231 and HBL100 cells (with downregulated hTERT) showed no c-myc binding, while mad1 binding was observed. In SK-BR-3 and BT-474 cells (with upregulated hTERT), the binding of mad1 became undetectable at 48 and $72 \mathrm{~h}$, respectively. MCF-7/HER2 cells demonstrated signals for all precipitations in non-irradiated and in $20 \mathrm{~Gy}$ irradiated cells at $96 \mathrm{~h}$, although a weaker signal was present for c-myc in non-irradiated cells and for mad1 in irradiated cells. As a control, the chromatin was immunoprecipitated with $\beta$-galactosidase antibodies. In these immunoprecipitates no hTERT sequences could be detected, demonstrating the specificity of the procedure. Input chromatin was positive for all samples tested.

HER2 and hTERT knockdown decreases the resistance of cancer cells to irradiation. The significance of HER2 and telomerase in cell viability and the resistance of breast cancer cells to irradiation was investigated after knockdown of HER2 or hTERT and subsequent irradiation. Cell viability was assessed by MTT assay.

As the dosage of $80 \mathrm{nM}$ of siRNA against HER2 was the most effective concerning the repression of hTERT/telomerase in SK-BR-3 cells, we used this dosage in order to investigate the role of HER2 in the increased radio-resistance of HER2positive cells (Fig. 5). We silenced HER2 expression by siRNA against HER2 and found a significant decrease (of almost $60 \%, 24$ h post-transfection) of cell viability in SK-BR-3 cells, which was higher $(\mathrm{F}=3620.3>>1, \mathrm{P}<0.05)$ than the decrease 
A

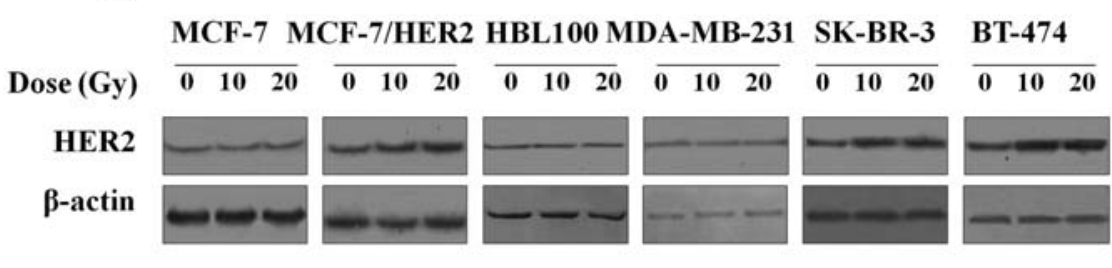

B

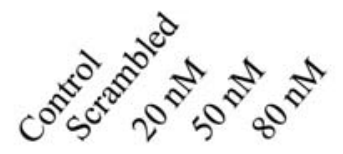

HER2

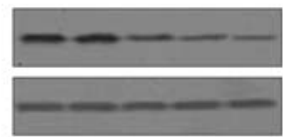

C

$\beta$-actin

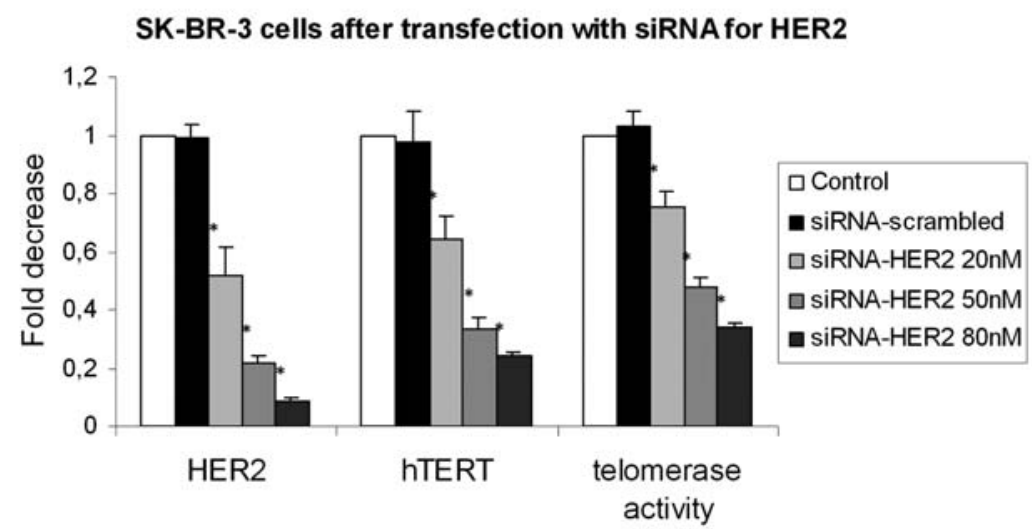

Figure 2. Effect of HER2 expression in the regulation of hTERT/telomerase. (A) Non-irradiated, 10 and $20 \mathrm{~Gy}$ irradiated cells were harvested at $24,48,72$ and $96 \mathrm{~h}$, lysed and $40 \mu \mathrm{g}$ of total protein were subjected to Western blot analysis of HER2 protein. Analysis for $\beta$-actin was performed to show equal loading. For simplicity, only 48-h blots are represented here. (B) Effect of HER2 knockdown on HER2 protein, as demonstrated by Western blot analysis and (C) on HER2 and hTERT mRNA expression and telomerase activity. Bars represent standard errors and asterisks designate statistically significant results $(\mathrm{P}<0.05)$.

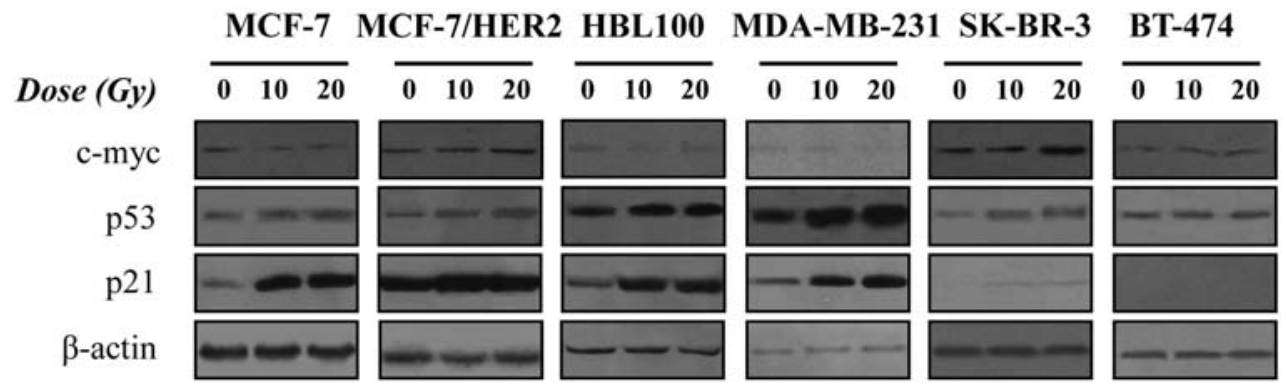

Figure 3. Effect of ionizing radiation on c-myc, p53 and p21 protein expression in MCF-7, MCF-7/HER2, MDA-MB-231, HBL100, SK-BR-3 and BT-474 breast cancer cells. For simplicity, only 48-h blots (non-irradiated, 10 and 20 Gy irradiated cells) are presented here. Western blot analysis for $\beta$-actin was performed to show equal loading.

caused by irradiation $(\mathrm{F}=222.7>>1, \mathrm{P}<0.05)$. Univariate 2 factor analysis of variance indicates that both examined factors (siRNA against HER2 and irradiation) have an even more significant effect on cell viability by reducing it by $80 \%, 24 \mathrm{~h}$ post-transfection $(\mathrm{F}=3869.5>>1, \mathrm{P}<0.05)$.

To demonstrate whether the increased radio-resistance of SK-BR-3 cells is mediated through hTERT/telomerase, we also knocked down hTERT. The effect of hTERT silencing in hTERT expression and telomerase activity is demonstrated in
Fig. 5B. As demonstrated in panel C, hTERT silencing in SK-BR-3 cells caused a significant reduction in cell viability in both non-irradiated and $10 \mathrm{~Gy}$ irradiated cells, but it was less than the one observed after HER2 knockdown. The analysis of variance yielded high F-statistics for both irradiation $(\mathrm{F}=87.5)$ and especially hTERT knock-down $(\mathrm{F}=567.1)$. Their combined effect was much stronger $(\mathrm{F}=669.8)$. The corresponding P-values were $<0.05$ threshold thus indicating statistically significant relationships. 


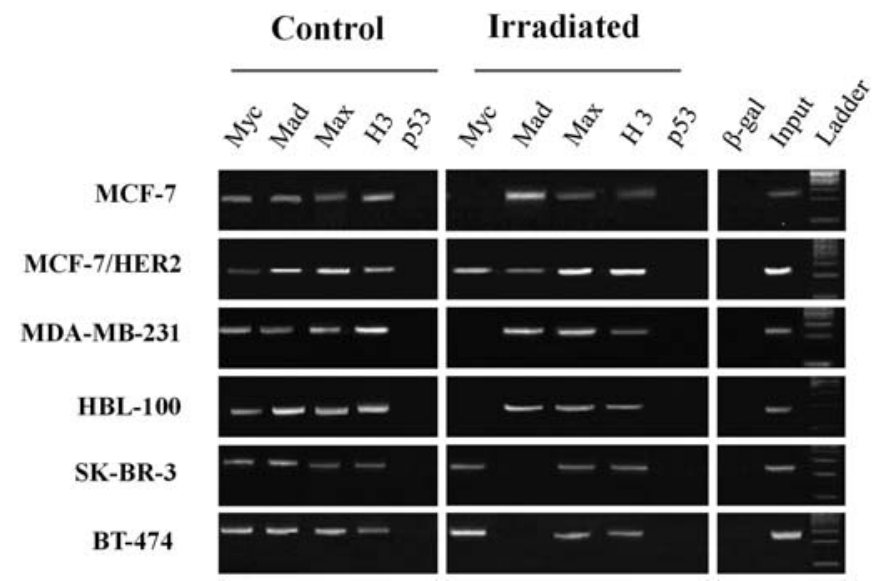

Figure 4. Chromatin immunoprecipitation analysis of hTERT promoter in irradiated and non-irradiated breast cancer cell lines. Differential c-myc/ $\max , \operatorname{mad} 1 / \max$ and $\mathrm{p} 53$ binding and histone $\mathrm{H} 3$ acetylation status in hTERT promoter in irradiated and non-irradiated MCF-7, MCF-7/HER2, MDA-MB231, HBL-100, SK-BR-3 and BT-474 cells is shown.

\section{Discussion}

Radiotherapy plays an important role in the management of breast cancer but the frequent occurrence of radiation-resistant tumors is a common clinical problem. Knowledge on the molecular mechanisms employed by tumor cells to evade the inhibitory activity of radiotherapy is essential for the design of novel treatment strategies aiming to improve the effectiveness of radiotherapy (2). In the present study, we investigated the role of HER2 and p53 status in the regulation of hTERT expression and telomerase activity in different breast cancer cell lines after ionizing irradiation.

We observed that MCF-7/HER2, SK-BR-3 and BT-474 (highly expressing HER2) cells demonstrated higher hTERT/ telomerase levels than MCF-7, MDA-MB-231 and HBL100 (weakly expressing HER2) cells. Following irradiation HER2positive cells further upregulated HER2, c-myc and hTERT expression. The observation that MCF-7/HER2 cells, differing from their parental MCF-7 cell line only in their HER2 status, demonstrated a different hTERT expression profile and telomerase activity upon irradiation, suggests the implication of HER2 in telomerase regulation. This is in accordance with previous findings, in which hTERT expression has been found to be increased in HER2-positive compared to HER2-negative breast tumor cell lines (18). HER2 is a highly characterized oncoprotein, involved in the control of diverse biological processes, such as proliferation, differentiation, migration and apoptosis (22). As a receptor tyrosine kinase related to the epidermal growth factor receptor, HER2 mediates tumor formation in breast, ovary, lung, stomach, colon, kidney,

A

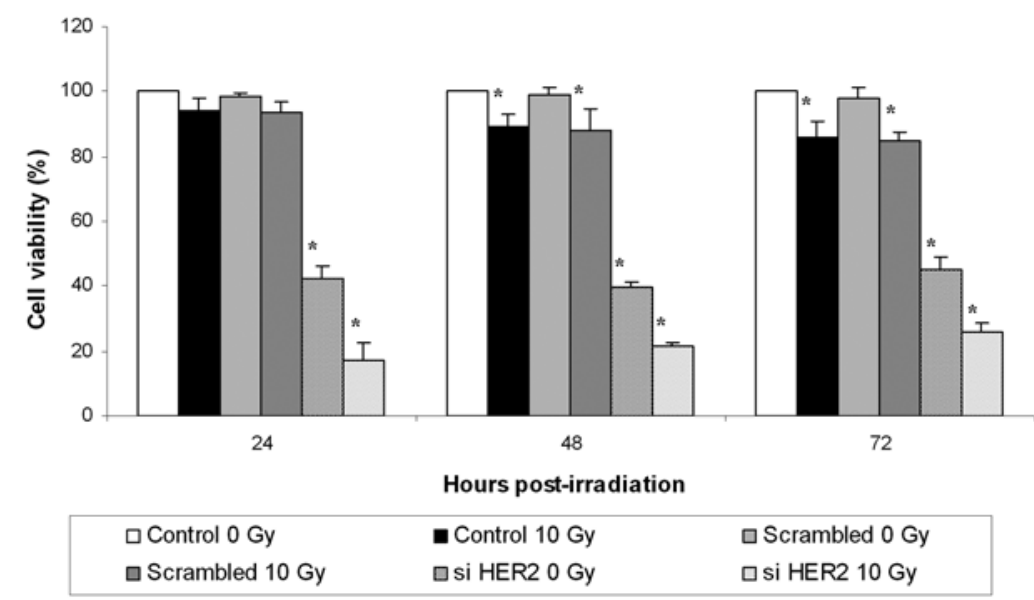

B

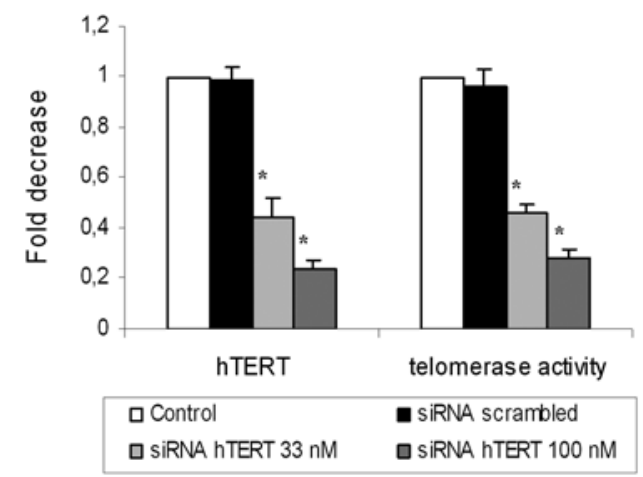

C

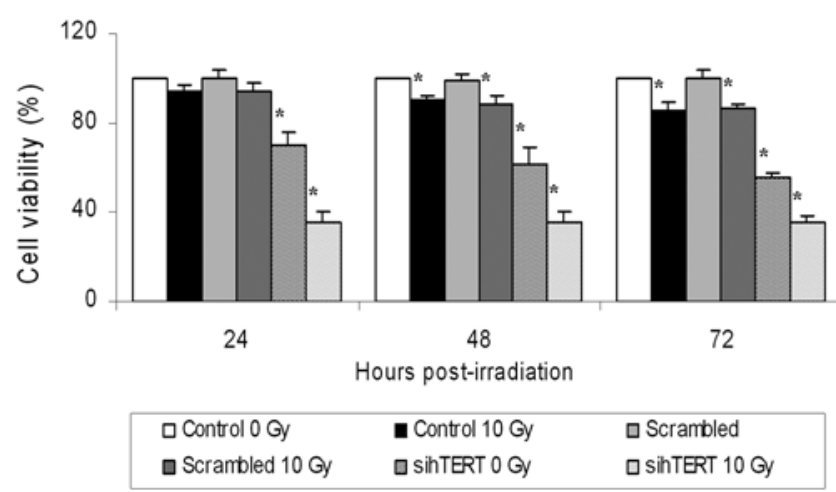

Figure 5. (A) SK-BR-3 cells were transfected with siRNA against HER2. Twenty-four hours post-transfection, cells were irradiated with a single 10 Gy dose and cell viability was assessed by MTT assay 24,48 and $72 \mathrm{~h}$ post-irradiation. Cell viability in non-transfected, non-irradiated cells was considered to be $100 \%$. Bars represent standard errors and asterisks designate statistically significant results $(\mathrm{P}<0.05)$. (B) SK-BR-3 cells were transfected with siRNA for hTERT. Increasing doses of siRNA for hTERT (33 and $100 \mathrm{nM}$ ) caused effective silencing of hTERT measured by real-time PCR and of telomerase activity, measured by TRAP assay. (C) SK-BR-3 cells were transfected with $33 \mathrm{nM}$ of siRNA for hTERT, irradiated with a single 10 Gy and measured for cell viability using MTT assay 24, 48 and $72 \mathrm{~h}$ post-irradiation. Bars represent standard errors and asterisks designate statistically significant results $(\mathrm{P}<0.05)$. 
bladder and salivary gland. It has been shown that HER2 overexpression accounts for 20 to $30 \%$ of human breast tumors and affects prognosis adversely $(23,24)$.

In addition to HER2 status, p53 status has also been reported to be correlated with the regulation of hTERT/ telomerase in human tumor cells. Overexpression of wild-type p53 has been shown to downregulate telomerase enzymatic activity in a number of cancer cell lines, independently of its effects on growth arrest and apoptosis. This has been attributed to transcriptional repression of hTERT by wild-type p53, since it was preceded by downregulation of hTERT mRNA through five Sp1 transcription factor-binding sites within the hTERT core promoter (25). Previously, it was shown that p53induced hTERT repression occurs through an indirect mechanism mediated by the $\mathrm{p} 21 / \mathrm{E} 2 \mathrm{~F}$ pathway (26). In the present study, we investigated p53 expression in both p53 wild-type (MCF-7, MCF-7/HER2 and HBL100) and p53 mutant (MDA-MB-231, SK-BR-3 and BT-474) cell lines, as well as the ability of wild and mutant $\mathrm{p} 53$ protein to induce p21 expression. We found that all cell lines expressed p53 which was upregulated after irradiation and induced p21 expression with the exception of BT-474 cells. The above findings suggest that p53-dependent induction of p21 could be responsible for the hTERT repression observed in MCF-7, HBL100 and MDA-MB-231 cells, but also for the relatively tardive upregulation of hTERT in MCF-7/HER2 cells. The low p21 expression in SK-BR-3 cells and the absence of expression in BT-474 cells, could be responsible for the steady hTERT upregulation after irradiation.

We then analyzed the occupancy of $h T E R T$ promoter by endogenous $\mathrm{p} 53$, c-myc, mad1 and max proteins, as well as the acetylation status of $\mathrm{H} 3$ histone, in irradiated and non-irradiated breast cancer cell lines. hTERT promoter was not detected at any p53 precipitate in accordance with recent observations showing that the repression of hTERT expression by endogenous p53 is indirect and mediated by p21 and the $\mathrm{Rb} / \mathrm{E} 2 \mathrm{~F}$ pathway (26). It is known that p53 acts as repressor in a vast number of genes $(27,28)$. Unlike transcriptional activation by $\mathrm{p} 53$, which is mediated through direct p53 binding to consensus sites in the regulatory regions of target genes (28), transcriptional repression is less well understood, since the promoters of repressed genes, usually do not contain consensus binding sites. We found for the first time, that hTERT upregulation was correlated with preferential fixing of myc/max complexes in the proximal E-box in the promoter region of hTERT gene in HER2-positive irradiated cells, while hTERT downregulation was correlated with fixing of mad1/max in HER2-negative cells. The critical role of c-myc and its network proteins in regulating $h T E R T$ promoter has been demonstrated by several studies in different types of cancer including breast cancer, lung cancer and leukemic cells (29-31).

It has been suggested that transactivation by myc, may involve recruitment of histone acetyltransferases (32), which consequently would result in increased histone acetylation at the promoter region. We did not observe loss, but a reduction of acetylated H3 signal in HER2-negative cell lines (MCF-7, MDA-MB-231 and HBL100), in which hTERT was downregulated and the inverse was observed for the HER2-positive MCF-7/HER2 cell line. These findings are in accordance with the relative litterature but we cannot exclude the possibility that the recruitment of histone deacetylases by mad repressor complexes to the $h T E R T$ promoter may also be taking place at cis-regulatory elements other than E-boxes.

In order to confirm the role of HER2 and hTERT in the radio-resistance of HER2-positive cells, we proceeded with silencing of both genes through transfection with liposomes and subsequent irradiation of HER2-positive SK-BR-3 cells. We observed that HER2 and hTERT knockdown caused significantly higher decrease in cell viability than the one caused by ionizing radiation. The combination of hTERT or HER 2 knockdown along with irradiation, increased significantly cell mortality, especially after HER2 silencing, suggesting that HER2 phenotype is important for the viability of HER2-positive cells, whose reduction could be attributed to the downregulation of target genes, such as telomerase.

In conclusion, we propose a model in which HER2 and p53 play an important role in the regulation of hTERT/ telomerase after irradiation in breast cancer cells, suggesting that wild-type p53, as well as two forms of mutant p53 (mutations R280K and R175H of MDA-MB-231 and SKBR-3 respectively), could be responsible for the downregulation of hTERT/telomerase upon irradiation, through an indirect mechanism, involving p21 induction. Also, HER2 overexpression, through proteins of the $\mathrm{myc} / \mathrm{mad} / \mathrm{max}$ network seemed to be a functional transcriptional regulator of hTERT expression in irradiated breast cancer cells. However, as hTERT/telomerase regulation is a complex process, further relative studies are needed. In order to verify our findings in the in vivo situation, we are already in the process of evaluating our results in fresh tissues obtained from breast cancer patients. Furthermore, our results point towards the potential use of small interference RNA technology against hTERT in HER2-positive breast cancers, targeting not only the inhibition of cancer cell proliferation but also the enhancement of their radio-sensitivity.

\section{References}

1. Feuer EJ, Wun LM, Boring CC, Flanders WD, Timmel MJ and Tong T: The lifetime risk of developing breast cancer. J Natl Cancer Inst 85: 892-897, 1993.

2. Gee JM and Nicholson RI: Expanding the therapeutic repertoire of epidermal growth factor receptor blockade: radiosensitization. Breast Cancer Res 5: 126-129, 2003.

3. Lichter AS and Lawrence TS: Recent advances in radiation oncology. N Engl J Med 332: 371-379, 1995.

4. Canman CE, Gilmer TM, Coutts S and Kastan M: Growth factor modulation of p53-mediated growth arrest versus apoptosis. Genes Dev 9: 600-611, 1995.

5. Kim NW, Piatyszek MA, Prowse KR, Harley CB, West MD, Ho PL, Coviello GM, Wright WE, Weinrich SL and Shay JW: Specific association of human telomerase activity with immortal cells and cancer. Science 266: 2011-2015, 1994.

6. Shay JW and Bacchetti S: A survey of telomerase activity in human cancer. Eur J Cancer 33: 787-791, 1997.

7. Pandita TK and Roti Roti JL: Role of telomerase in radiocurability. Oncol Rep 10: 263-270, 2003.

8. Asker C, Wiman KG and Selivanova G: p53-induced apoptosis as a safeguard against cancer. Biochem Biophys Res Commun 265: 1-6, 1999.

9. Xu D, Wang Q, Gruber A, Björkholm M, Chen Z, Zaid A, Selivanova G, Peterson C, Wiman KG and Pisa P: Downregulation of telomerase reverse transcriptase mRNA expression by wild type p53 in human tumor cells. Oncogene 19: 5123-5133, 2000 . 
10. Counter CM, Avilion AA, LeFeuvre CE, Stewart NG, Greider CW, Harley CB and Bacchetti S: Telomere shortening associated with chromosome instability is arrested in immorta cells which express telomerase activity. EMBO J 11: 1921-1929, 1992.

11. Greider CW and Blackburn EH: A telomeric sequence in the RNA of Tetrahymena telomerase required for telomere repeat synthesis. Nature 337: 331-337, 1989.

12. Cohen SB, Graham ME, Lovrecz GO, Bache N, Robinson PJ and Reddel RR: Protein composition of catalytically active human telomerase from immortal cells. Science 315: 1850-1853, 2007.

13. Meyerson M, Counter CM, Eaton EN, Ellisen LW, Steiner P, Caddle SD, Ziaugra L, Beijersbergen RL, Davidoff MJ, Liu Q, Bacchetti S, Haber DA and Weinberg RA: hEST2, the putative human telomerase catalytic subunit gene, is up-regulated in tumor cells and during immortalization. Cell 90: 785-795, 1997.

14. Nakamura TM, Morin GB, Chapman KB, Weinrich SL, Andrews WH, Lingner J, Harley CB and Cech TR: Telomerase catalytic subunit homologs from fission yeast and human. Science 277: 955-959, 1997.

15. Bodnar AG, Ouellette M, Frolkis M, Holt SE, Chiu CP Morin GB, Harley CB, Shay JW, Lichtsteiner S and Wright WE: Extension of life-span by introduction of telomerase into normal human cells. Science 279: 349-352, 1998.

16. Sawant SG, Gregoire V, Dhar S, Umbricht CB, Cvilic S, Sukumar S and Pandita TK: Telomerase activity as a measure for monitoring radiocurability of tumor cells. FASEB J 13: 1047-1054, 1999.

17. Kyo S and Inoue M: Complex regulatory mechanisms of telomerase activity in normal and cancer cells: how can we apply them for cancer therapy? Oncogene 21: 688-697, 2002.

18. Goueli BS and Janknecht R: Upregulation of the catalytic telomerase subunit by the transcription factor ER81 and oncogenic HER2/Neu, Ras, or Raf. Mol Cell Biol 24: 25-35, 2004 .

19. Ko LJ and Prives C: p53: puzzle and paradigm. Genes Dev 10: 1054-1072, 1996.

20. Satra M, Tsougos I, Papanikolaou V, Theodorou K, Kappas C and Tsezou A: Correlation between radiation-induced telomerase activity and human telomerase reverse transcriptase mRNA expression in HeLa cells. Int J Radiat Biol 82: 401-409, 2006.
21. Tsezou A, Oikonomou P, Kollia P, Mademtzis I, Kostopoulou E, Messinis I and Vamvakopoulos N: The role of human telomerase catalytic subunit mRNA expression in cervical dysplasias. Exp Biol Med 230: 263-270, 2005.

22. Liang K, Lu Y, Jin W, Ang KK, Milas L and Fan Z: Sensitization of breast cancer cells to radiation by trastuzumab. Mol Cancer Ther 2: 1113-1120, 2003.

23. Hynes NE and Stern DF: The biology of erbB-2/neu/HER-2 and its role in cancer. Biochim Biophys Acta 1198: 165-184, 1994.

24. Yarden Y: Biology of HER2 and its importance in breast cancer. Oncology 61: 1-13, 2001.

25. Kusumoto M, Ogawa T, Mizumoto $K$, Ueno $H$, Niiyama $H$, Sato N, Nakamura M and Tanaka M: Adenovirus-mediated p53 gene transduction inhibits telomerase activity independent of its effects on cell cycle arrest and apoptosis in human pancreatic cncer cells. Clin Cancer Res 5: 2140-2147, 1999.

26. Shats I, Myliavsky M, Tang X, Stambolsky P, Erez N, Brosh R, Kogan I, Braunstein I, Tzukerman M, Ginsberg D and Rotter V: p53-dependent down-regulation of telomerase is mediated by p21waf1. J Biol Chem 279: 50976-50985, 2004.

27. Zhao R, Gish K, Murphy M, Yin Y, Notterman D, Hoffman WH, Tom E, Mack DH and Levine AJ: Analysis of p53-regulated gene expression patterns using oligonucleotide arrays. Genes Dev 14: 981-993, 2000

28. el-Deiry WS, Kern SE, Pietenpol JA, Kinzler KW and Vogelstein B: Definition of a consensus binding site for p53. Nat Genet 1: 45-49, 1992.

29. Cosgrave N, Hill AD and Young LS: Growth factor-dependent regulation of survivin by c-myc in human breast cancer. J Mol Endocrinol 37: 377-390, 2006.

30. Fujiki T, Miura T, Maura M, Shiraishi H, Nishimura S, Imada Y, Uehara N, Tashiro K, Shirahata S and Katakura Y: TAK1 represses transcription of the human telomerase reverse transcriptase gene. Oncogene 26: 5258-5266, 2007.

31. Xu D, Popov N, Hou M, Wang O, Björkholm M, Gruber A, Menkel AR and Henriksson M: Switch from Myc/Max to Mad1/Max binding and decrease in histone acetylation at the telomerase reverse transcriptase promoter during differentiation of HL60 cells. Proc Natl Acad Sci USA 98: 3826-3831, 2001.

32. Cole MD and McMahon SB: The Myc oncoprotein: a critical evaluation of transactivation and target gene regulation. Oncogene 18: 2916-2924, 1999. 